\title{
FUCHSIAN GROUPS AND ALGEBRAIC NUMBER FIELDS
}

\author{
BY \\ P. L. WATERMAN ${ }^{1}$ AND C. MACLACHLAN
}

\begin{abstract}
Given the signature of a finitely-generated Fuchsian group, we find the minimal extension of the rationals for which there is a Fuchsian group having the required signature, whose matrix entries lie in this field.
\end{abstract}

1. Let $\Gamma$ be a Fuchsian group, i.e., a discrete subgroup of $\operatorname{PSL}(2, R)$. If it is finitely generated and nonelementary it has a presentation of the form:

$$
\begin{array}{ll}
\text { Generators: } & a_{1}, b_{1}, \ldots, a_{g}, b_{g}, e_{1}, \ldots, e_{r}, p_{1}, \ldots, p_{s}, h_{1}, \ldots, h_{t} \\
\text { Relations: } & \prod_{j=1}^{g}\left[a_{j}, b_{j}\right] \prod_{i=1}^{r} e_{i} \prod_{k=1}^{s} p_{k} \prod_{l=1}^{t} h_{l}=1, \\
& e_{i}^{m_{i}}=1 \quad(i=1, \ldots, r), m_{i} \geqslant 2,
\end{array}
$$

with

$$
2(g-1)+s+t+\sum_{i=1}^{r}\left(1-\frac{1}{m_{i}}\right)>0 .
$$

The integers appearing in this presentation- $\left(g ; m_{1}, m_{2}, \ldots, m_{r} ; s ; t\right)$-form the signature of the group. Furthermore, for any collection of such integers, there exists a Fuchsian group with the corresponding presentation and signature. The elements of a Fuchsian group can be represented by $2 \times 2$ matrices of determinant one up to a factor of \pm 1 , so the field of definition of a Fuchsian group is well defined. In this paper we examine the smallest fields of definition among groups with the same signature. For a group $\Gamma$ with presentation at (1), it is clear that the field of definition must contain

$$
Q\left(\cos \frac{\pi}{m_{1}}, \cos \frac{\pi}{m_{2}}, \ldots, \cos \frac{\pi}{m_{r}}\right) .
$$

It turns out (see \$2) that this field is a field of definition for a group $\Gamma$ with presentation at (1) unless $\Gamma$ is a cocompact triangle group. In contrast, our main result (Theorem 3 ) is that that is not the case for all but a finite number of signatures of cocompact triangle groups.

Received by the editors January 30, 1984.

1980 Mathematics Subject Classification. Primary 30F35, 20H10, 10D07; Secondary 32G15.

${ }^{1}$ The first author acknowledges receipt of a S.R.C. grant. 
Since PSL $(2, R)$ can be identified with the component $\operatorname{PGL}_{0}(2, R)$ of the identity in $\operatorname{PGL}(2, R)$, elements of Fuchsian groups can also be represented by $2 \times 2$ matrices with nonzero determinant. Similar questions to those above are considered for fields of definition in this case in $§ 6$.

2. Let $\sigma=\left(g ; m_{1}, m_{2}, \ldots, m_{r} ; s ; t\right)$ be the signature of a nonelementary finitelygenerated Fuchsian group.

Definition. The signature $\sigma$ is said to have a representative in the field $K$ if there exists a Fuchsian group $\Gamma$ of signature $\sigma$ such that $\Gamma<\operatorname{PSL}(2, K)$.

As noted above,

$$
K>Q\left(\cos \frac{\pi}{m_{1}}, \cos \frac{\pi}{m_{2}}, \ldots, \cos \frac{\pi}{m_{r}}\right) .
$$

Mennicke [14] showed that $(g ;-; 0 ; 0)$ had a representative in $Q(\sqrt{3})$, and Magnus, [13] showed that it had a representative in $Q$ by exhibiting an explicit rational $2 \times 2$ presentation of a group with signature $(1 ; 2 ; 0 ; 0)$. Millington [15] has observed that groups with any signature of the form $(g ; 2,2, \ldots, 2,3,3, \ldots, 3 ; s ; 0)$, where $s>0$, exist as subgroups of the modular group and so such signatures have representatives in $Q$. Similar results will follow from recent work of Kulkarni $[2,3,4,10]$ on characterising subgroups of finite index in a given Fuchsian group. Lehner and Newman $[11,12]$ have considered the cases $\left(0 ; m_{1}, \ldots, m_{r} ; s ; t\right)$, where $r+s+t=3$, $r<3$.

More significantly, Takeuchi [16] uses a method of deforming a group $\Gamma$ in its representation space to prove that the signature $(g ; 2,2, \ldots, 2,3,3, \ldots, 3 ; 0 ; 0)$ has representatives in $Q$. We will now sketch his method and indicate how it can be generalised to all signatures except those of cocompact triangle groups. Let $\Gamma$ be a Fuchsian group with signature of the form $(g ; 2,2, \ldots, 2,3,3, \ldots, 3 ; 0 ; 0)$. For a set of generators of $\Gamma$, let $\mathscr{R}(\Gamma)$ denote the space of homomorphisms $\phi: \Gamma \rightarrow \operatorname{PSL}(2, R)$ topologised by the images of the generators. The subspace $\mathscr{R}_{0}(\Gamma)$ of all isomorphisms with discrete image group was proved by Weil [18] to be open in $\mathscr{R}(\Gamma)$. Takeuchi showed that one can perturb $\phi \in \mathscr{R}_{0}(\Gamma)$ such that the perturbed generators lie in $\operatorname{PSL}(2, Q)$. For small enough perturbations the result follows. A suitable generalisation of Weil's theorem [1] exists for all nonelementary finitely-generated Fuchsian groups. Further, Takeuchi's argument can be extended to study representatives of $\sigma=\left(g ; m_{1}, m_{2}, \ldots, m_{r} ; s ; t\right)$ in $\operatorname{PSL}(2, K)$, where

$$
K=Q\left(\cos \frac{\pi}{m_{1}}, \cos \frac{\pi}{m_{2}}, \ldots, \cos \frac{\pi}{m_{r}}\right),
$$

except in those cases corresponding to cocompact triangle groups, i.e., where $g=s=t=0$ and $r=3$. (For details see [17].) Takeuchi further modified his argument to show that the perturbation can be organised so that one element of the perturbed group has trace distinct from all traces in the original group. Now the Teichmüller space of $\Gamma, \mathscr{T}(\Gamma)$, is the quotient of $\mathscr{R}_{0}(\Gamma)$ under conjugation by elements of $\operatorname{PGL}(2, R)$. If $\mathscr{T}_{K}(\Gamma)$ represents the subspace corresponding to groups whose elements lie in $\operatorname{PSL}(2, K)$, then Takeuchi's trace argument shows that $\mathscr{T}_{Q}(\Gamma)$ 
is dense in $\mathscr{T}(\Gamma)$. This argument can also be extended to any finitely-generated Fuchsian group whose Teichmüller space has positive dimension (see [17]).

THEOREM 1. If $\Gamma$ has signature $\left(g ; m_{1}, m_{2}, \ldots, m_{r} ; s ; t\right)$ and is not a cocompact triangle group, then $\mathscr{T}_{K}(\Gamma)$ is dense in $\mathscr{T}(\Gamma)$, where

$$
K=Q\left(\cos \frac{\pi}{m_{1}}, \cos \frac{\pi}{m_{2}}, \ldots, \cos \frac{\pi}{m_{r}}\right) .
$$

3. We now consider cocompact triangle groups, whose signatures $(0 ; l, m, n ; 0 ; 0)$ we shorten to $(l, m, n)$. Denote the image of $X \in \operatorname{SL}(2, R)$ in $\operatorname{PSL}(2, R)$ by $\bar{X}$. It is clear from (1) that a group with signature $(l, m, n)$ can be generated by two elements, and we make use of the following result of Knapp [9] on such groups.

TheOrem. A subgroup $\Gamma$ of $\operatorname{PSL}(2, R)$ is a Fuchsian group of signature $(l, m, n)$ if and only if there exist elements $A, B \in \operatorname{SL}(2, R)$ such that $\Gamma=\langle\bar{A}, \bar{B}\rangle$ and

$$
\operatorname{tr} A=-2 \cos \frac{\pi}{l}, \quad \operatorname{tr} B=-2 \cos \frac{\pi}{m}, \quad \operatorname{tr} A B=-2 \cos \frac{\pi}{n} .
$$

THEOREM 2. $\sigma=(l, m, n)$ has a representative in $K$, where

$$
Q\left(\cos \frac{\pi}{l}, \cos \frac{\pi}{m}, \cos \frac{\pi}{n}\right)<K<R,
$$

if and only if there is a solution in $K$ of the equation

$$
\left(1-\cos ^{2} \frac{\pi}{l}\right) x^{2}+y^{2}=-d
$$

where

$$
d=1-\cos ^{2} \frac{\pi}{l}-\cos ^{2} \frac{\pi}{m}-\cos ^{2} \frac{\pi}{n}-2 \cos \frac{\pi}{l} \cos \frac{\pi}{m} \cos \frac{\pi}{n} .
$$

Proof. By conjugating in $\operatorname{SL}(2, K)$, we can assume that

$$
\begin{gathered}
A=\left(\begin{array}{cc}
0 & \lambda \\
-1 / \lambda & -2 \cos (\pi / l)
\end{array}\right), \\
B=T C T^{-1} \quad \text { where } T=\left(\begin{array}{ll}
1 & \alpha \\
0 & 1
\end{array}\right),
\end{gathered}
$$

and

$$
C=\left(\begin{array}{cc}
0 & \mu \\
-1 / \mu & -2 \cos (\pi / m)
\end{array}\right)
$$

with $\alpha, \lambda, \mu \in K$ and $\lambda, \mu \neq 0$. The equation $\operatorname{tr} A B=-2 \cos \frac{\pi}{n}$ becomes

$$
\alpha^{2}+2 \alpha\left(\lambda \cos \frac{\pi}{l}-\mu \cos \frac{\pi}{m}\right)+\left(\lambda^{2}+\mu^{2}-2 \lambda \mu\left(2 \cos \frac{\pi}{l} \cos \frac{\pi}{m}+\cos \frac{\pi}{n}\right)\right)=0 \text {. }
$$

For a solution of this quadratic in $K$, its discriminant

$$
4\left(\cos ^{2} \frac{\pi}{l}-1\right) \lambda^{2}+8\left(\cos \frac{\pi}{l} \cos \frac{\pi}{m}+\cos \frac{\pi}{n}\right) \lambda \mu+4\left(\cos ^{2} \frac{\pi}{m}-1\right) \mu^{2}
$$

must be a square in $K$. Completing the square and using the fact that $\mu \neq 0$, then gives equation (3). 
From (2) it follows that $1 / l+1 / m+1 / n<1$ and, hence, that $d<0$ (see (4) below). Thus

Corollary. $\sigma=(l, m, n)$ has a representative in $Q\left(\cos \frac{\pi}{l}, \cos \frac{\pi}{m}, \cos \frac{\pi}{n}, \sqrt{-d}\right)$.

Since this field is an extension of degree at most 2 over the minimal possible field $Q\left(\cos \frac{\pi}{l}, \cos \frac{\pi}{m}, \cos \frac{\pi}{n}\right)$, it remains to determine whether or not $\sigma=(l, m, n)$ has a representative in this minimal possible field. Our main result is the following:

THEOREM 3. Except for finitely many triples $(l, m, n), \sigma=(l, m, n)$ does not have a representative in $Q\left(\cos \frac{\pi}{l}, \cos \frac{\pi}{m}, \cos \frac{\pi}{n}\right)$.

Suppose that $\sigma=(l, m, n)$ did have a representative in the minimal field so that (3) has a solution $(x, y)$ there. Now $Q\left(\cos \frac{\pi}{l}, \cos \frac{\pi}{m}, \cos \frac{\pi}{n}\right)$ is a Galois extension of $Q$ and every Galois automorphism of it is a restriction of a Galois automorphism of the cyclotomic field $Q\left(e^{i \pi / N}\right)$, where $N=1$. c.m. $\{l, m, n\}$. Thus every Galois automorphism $\sigma$ of $Q\left(\cos \frac{\pi}{l}, \cos \frac{\pi}{m}, \cos \frac{\pi}{n}\right) \mid Q$ is determined by

$$
\sigma\left(\cos \frac{\pi}{l}\right)=\cos \frac{s \pi}{l}, \quad \sigma\left(\cos \frac{\pi}{m}\right)=\cos \frac{s \pi}{m}, \quad \sigma\left(\cos \frac{\pi}{n}\right)=\cos \frac{s \pi}{n}
$$

for some integer $s$ with $(s, 2 \operatorname{lm} n)=1$. Thus if

$d(x)=1-\cos ^{2} \frac{x \pi}{l}-\cos ^{2} \frac{x \pi}{m}-\cos ^{2} \frac{x \pi}{n}-2 \cos \frac{x \pi}{l} \cos \frac{x \pi}{m} \cos \frac{x \pi}{n}, \quad x \in R$.

Then $\sigma(d)=d(s)$ for $s$ with $(s, 2$ lmn $)=1$. Applying $\sigma$ to equation (3) shows that

$$
\left(1-\cos ^{2} \frac{s \pi}{l}\right) x_{1}^{2}+y_{1}^{2}=-d(s)
$$

has a solution in $Q\left(\cos \frac{\pi}{l}, \cos \frac{\pi}{m}, \cos \frac{\pi}{n}\right)$ for all $s$ such that $(s, 2 \operatorname{lmn})=1$. If $d(s)>0$, this clearly cannot have a solution in any real subfield. Thus Theorem 3 will follow once we have established

THEOREM 4. Let $(l, m, n)$ be positive integers such that $1 / l+1 / m+1 / n<1$. Then, except for finitely many triples $(l, m, n)$, there exists an integer $s$ with $(s, 2 \operatorname{lmn})$ $=1$ such that $d(s)>0$.

4. This section is devoted to the proof of Theorem 4. The general technique is to find an $x \in R$ such that $d(x)>0$ and then to argue that there exists an integer $s$ with $(s, 2 \operatorname{lm} n)=1$ sufficiently close to $x$ so that $d(s)>0$. To obtain estimates of "sufficiently close" we use the following result.

LEMMA. If $\rho(n)=n^{1 / \log \log n}$, then, for sufficiently large $n$, and all integers $l, \alpha$ with $(\alpha, l)=1$ and $0<\alpha<l$, there exists in every interval of length $l \rho(n)$ an integer s such that $s \equiv \alpha(\bmod l)$ and $(s, n)=1$.

Proof. For sufficiently large $n, \rho$ is an increasing function and so it suffices to prove the result in the cases where $(l, n)=1$. For suppose it is true in these cases for all $n \geqslant N_{0}$. Let $N_{1}$ be such that $\rho\left(N_{1}\right) \geqslant N_{0}$. Then we claim it will be true in general for all $n \geqslant N_{1}$. For suppose $l$ is given and $n=n_{1} n^{\prime}$, where $\left(n^{\prime}, l\right)=1$, and if a prime $p$ divides $n_{1}$ then it divides $l$. So, if $n^{\prime} \geqslant N_{0}$, in any interval of length $l \rho(n) \geqslant l \rho\left(n^{\prime}\right)$ 
there exists an $s \equiv \alpha(\bmod l)$ with $\left(s, n^{\prime}\right)=1$, and so $(s, n)=1$. If $n^{\prime}<N_{0}$, then in any interval of length $l \rho(n) \geqslant l \rho\left(N_{1}\right) \geqslant l N_{0}>l n^{\prime}$, there exists an $s \equiv \alpha(\bmod l)$ with $\left(s, n^{\prime}\right)=1$, and so $(s, n)=1$.

Thus, we assume $(l, n)=1$. Let $p_{1}, p_{2}, \ldots, p_{r}$ be the prime divisors of $n$. Let $m_{i}$ denote the least positive solution of $m \equiv \alpha(\bmod l)$ and $m \equiv 0\left(\bmod p_{i}\right)$. Similarly, let $m_{i_{1} i_{2} \ldots}$ denote the least positive solution of $m \equiv \alpha(\bmod l)$ and $m \equiv 0\left(\bmod p_{i_{1}} p_{i_{2}}\right.$ $\cdots)$. Furthermore, if $f(\alpha, l, n ; x)$ denotes the number of positive integers $m \leqslant x$ such that $m \equiv \alpha(\bmod l)$ and $(m, n)=1$, then, by the Inclusion-Exclusion Theorem [8], for positive $x$,

$$
\begin{aligned}
f(\alpha, l, n ; x)= & {\left[\frac{x-\alpha}{l}+1\right]-\sum_{i}\left[\frac{x-m_{i}}{l p_{i}}+1\right]+\sum_{p_{i}<p_{j}}\left[\frac{x-m_{i j}}{l p_{i} p_{j}}+1\right] } \\
& -\cdots+(-1)^{r}\left[\frac{x-m_{1,2, \ldots, r}}{l p_{1} p_{2} \cdots p_{r}}+1\right] \\
= & \frac{x}{l}\left(1-\sum \frac{1}{p_{i}}+\sum \frac{1}{p_{i} p_{j}}-\cdots\right)+E=\frac{x}{l} \frac{\phi(n)}{n}+E
\end{aligned}
$$

where $|E| \leqslant 1+r+r(r-1) / 2+\cdots=2^{r} \leqslant d(n)$ and, here, $d(n)$ is the number of positive divisors of $n$. Hence, in any interval of length $t$ there are at least $t \phi(n) / l n-2 d(n)$ integers $s \equiv \alpha(\bmod l)$ such that $(s, n)=1$. In particular, in any interval of length exceeding $2 l(n / \phi(n)) d(n)$, there is at least one such integer. Now for any $\varepsilon>0$, the following two inequalities hold for large enough $n$ (see e.g. [8]):

$$
\begin{aligned}
d(n) & <2^{(1+\varepsilon)(\log n / \log \log n)} \\
n / \phi(n) & <(1+\varepsilon) e^{\gamma} \log \log n \quad(\gamma \text { : Euler's constant }) .
\end{aligned}
$$

Thus, for large enough $n$, we have

$$
(2 n / \phi(n)) d(n)<e^{\log n / \log \log n}=\rho(n) .
$$

It will be convenient to use the following factorisations of $d(x)$ which are obtained from the double angle formulae.

$$
\begin{aligned}
d(x)= & -4 \cos \frac{\pi}{2}\left(-\frac{x}{l}+\frac{x}{m}+\frac{x}{n}\right) \cos \frac{\pi}{2}\left(\frac{x}{l}-\frac{x}{m}+\frac{x}{n}\right) \\
& \cdot \cos \frac{\pi}{2}\left(\frac{x}{l}+\frac{x}{m}-\frac{x}{n}\right) \cos \frac{\pi}{2}\left(\frac{x}{l}+\frac{x}{m}+\frac{x}{n}\right) \\
= & -\left(\cos \frac{x \pi}{l}+\cos x \pi\left(\frac{1}{m}-\frac{1}{n}\right)\right)\left(\cos \frac{x \pi}{l}+\cos x \pi\left(\frac{1}{m}+\frac{1}{n}\right)\right) .
\end{aligned}
$$

Proof of Theorem 4. Assume that $l \leqslant m \leqslant n$ and $n$ is large enough to apply the above lemma. Let $\rho=\rho\left(2 n^{3}\right) \geqslant \rho(2 l m n)$. The proof splits into several cases depending on the relative sizes of $l, m, n$.

Case I. $(1 / l+1 / m)^{2} \leqslant 2 / n \rho$.

Let $s$ be the largest integer relatively prime to $2 \operatorname{lm} n$ and satisfying

$$
s(1 / l+1 / m+1 / n)<1,
$$

and let $s^{\prime}$ be the next integer $>s$ also relatively prime to $2 \operatorname{lm} n$. From (4), $d\left(s^{\prime}\right)>0$ if

$$
s^{\prime}\left(\frac{1}{l}+\frac{1}{m}+\frac{1}{n}\right)<3 \text { and } s^{\prime}\left(\frac{1}{l}+\frac{1}{m}-\frac{1}{n}\right)<1 .
$$


Now

$$
\begin{aligned}
s^{\prime}\left(\frac{1}{l}+\frac{1}{m}-\frac{1}{n}\right) & <(s+\rho)\left(\frac{1}{l}+\frac{1}{m}-\frac{1}{n}\right) \\
& <\left(\left(\frac{1}{l}+\frac{1}{m}+\frac{1}{n}\right)^{-1}+\rho\right)\left(\frac{1}{l}+\frac{1}{m}-\frac{1}{n}\right) \\
& =\left(\frac{1}{l}+\frac{1}{m}+\frac{1}{n}\right)^{-1}\left(\frac{1}{l}+\frac{1}{m}-\frac{1}{n}+\rho\left(\frac{1}{l}+\frac{1}{m}\right)^{2}-\frac{\rho}{n^{2}}\right) \\
& <\left(\frac{1}{l}+\frac{1}{m}+\frac{1}{n}\right)^{-1}\left(\frac{1}{l}+\frac{1}{m}-\frac{1}{n}+\frac{2}{n}\right)=1
\end{aligned}
$$

and

$$
\begin{aligned}
s^{\prime}\left(\frac{1}{l}+\frac{1}{m}+\frac{1}{n}\right) & =s^{\prime}\left(\frac{1}{l}+\frac{1}{m}-\frac{1}{n}\right)+\frac{2 s^{\prime}}{n} \\
& <1+\frac{2}{n}(s+\rho)<1+\frac{2}{n}\left(\frac{n}{3}+\frac{n}{2}\right)<3 .
\end{aligned}
$$

Case II. $(1 / l+1 / m)^{2} \geqslant 2 / n \rho, l \rho / m \geqslant 1 / 12, \rho / l \leqslant c(c$ a constant $)$.

For $r \in Z$ we deduce from (5) that

$$
d\left(\frac{r}{1 / l+1 / m}\right)=\left[\begin{array}{l}
\left(1-\cos \frac{r l m}{n(l+m)} \pi\right)\left(\cos \frac{r l m}{n(l+m)} \pi-\cos \frac{2 r l}{l+m} \pi\right), \\
-\left(1+\cos \frac{r l m}{n(l+m)} \pi\right)\left(\cos \frac{r l m}{n(l+m)} \pi+\cos \frac{2 r l}{l+m} \pi\right), \\
r \text { even. }
\end{array}\right.
$$

Now let

$$
r=\frac{n(l+m)}{l m} \cdot \frac{1}{3}+E
$$

where $E$ is chosen so that $r$ is odd and $|E| \leqslant 1$. Thus

$$
\begin{gathered}
d\left(\frac{r+e}{1 / l+l / m}\right)=\left[1-\cos \left(\frac{\pi}{3}+\frac{\operatorname{lm}(E+e)}{n(l+m)} \pi\right)\right] \\
\cdot\left[\cos \left(\frac{\pi}{3}+\frac{\operatorname{lm}(E+e)}{n(l+m)} \pi\right)-\cos \left(\frac{2 n \pi}{3 m}+\frac{2(E+e) l}{l+m} \pi\right)\right], \quad e \text { even; } \\
d\left(\frac{r+\delta}{1 / l+1 / m}\right)=-\left[1+\cos \left(\frac{\pi}{3}+\frac{\operatorname{lm}(E+\delta)}{n(l+m)} \pi\right)\right] \\
\quad \cdot\left[\cos \left(\frac{\pi}{3}+\frac{\operatorname{lm}(E+\delta)}{n(l+m)} \pi\right)+\cos \left(\frac{2 n \pi}{3 m}+\frac{2(E+\delta) l}{l+m} \pi\right)\right], \quad \delta= \pm 1 ; \\
d\left(\frac{2 r}{1 / l+1 / m}\right)=-\left[1+\cos \left(\frac{2 \pi}{3}+\frac{2 \ln E}{n(l+m)} \pi\right)\right] \\
\cdot\left[\cos \left(\frac{2 \pi}{3}+\frac{2 \ln E}{n(l+m)} \pi\right)+\cos \left(\frac{4 n \pi}{3 m}+\frac{4 E l}{l+m} \pi\right)\right] .
\end{gathered}
$$


The proof of Case II now splits into several subcases and we use the notation $y_{\bmod 2}$ for that unique number congruent to $y(\bmod 2)$ and lying in the interval $(-1,1]$.

$$
\left|\left(\frac{2 n}{3 m}+\frac{2 E l}{l+m}-1\right)_{\bmod 2}\right| \leqslant \frac{5}{8} \text { and } \frac{l m}{n(l+m)} \leqslant \frac{1}{48} .
$$

Then

$$
\begin{aligned}
& d\left(\frac{r}{1 / l+1 / m}\right) \geqslant\left[1-\cos \pi\left(\frac{1}{3}-\frac{1}{48}\right)\right]\left[\cos \pi\left(\frac{1}{3}+\frac{1}{48}\right)-\cos \pi\left(1+\frac{5}{8}\right)\right] \\
&=\left(1-\cos \frac{5 \pi}{16}\right)\left(\cos \frac{17 \pi}{48}-\cos \frac{18 \pi}{48}\right)=d_{1}>0, \\
& \frac{3}{16} \leqslant\left|\left(\frac{2 n}{3 m}+\frac{2 E l}{l+m}\right)_{\bmod 2}\right| \leqslant \frac{3}{8} \text { and } \frac{l m}{n(l+m)} \leqslant \frac{1}{96} .
\end{aligned}
$$

Then

$$
\begin{gathered}
d\left(\frac{2 r}{1 / l+1 / m}\right) \geqslant\left[1+\cos \pi\left(\frac{2}{3}+\frac{1}{48}\right)\right]\left[-\cos \pi\left(\frac{2}{3}-\frac{1}{48}\right)-\cos \frac{3 \pi}{8}\right] \\
=d_{1}>0 . \\
\left|\left(\frac{2 n}{3 m}+\frac{2 E l}{l+m}\right)_{\bmod 2}\right| \leqslant \frac{3}{16}, \quad \frac{l m}{n(l+m)} \leqslant \frac{1}{96}, \quad \frac{2 l}{l+m} \geqslant \frac{17}{24} .
\end{gathered}
$$

Choose $\delta= \pm 1$ so that

(IV)

$$
\begin{gathered}
d\left(\frac{r+\delta}{1 / l+1 / m}\right) \geqslant-\left[1+\cos \pi\left(\frac{1}{3}+\frac{1}{48}\right)\right]\left[\cos \pi\left(\frac{1}{3}-\frac{1}{48}\right)+\cos \frac{17 \pi}{24}\right] \\
=\left[1+\cos \frac{17 \pi}{48}\right]\left[\cos \frac{14 \pi}{48}-\cos \frac{15 \pi}{48}\right]=d_{2}>0 . \\
\mid\left(\frac{2 n}{3 m}+\frac{2 E l}{l+m}\right)_{\bmod 2} \leqslant \frac{3}{16}, \quad \frac{l m}{n(l+m)} \leqslant \frac{1}{9(17+12 \rho)}, \\
\frac{2 l}{l+m} \leqslant \frac{17}{24}, \quad \frac{l \rho}{m} \geqslant \frac{1}{12} .
\end{gathered}
$$

Thus

$$
\frac{2}{1+12 \rho} \leqslant \frac{2 l}{l+m} \leqslant \frac{17}{24} .
$$

Hence, for some even $e$, with $|e|<\frac{3}{16}(1+12 \rho)+2$, we have

$$
\left|\left(\frac{2 n}{3 m}+\frac{2 E l}{l+m}+\frac{2 e l}{l+m}-1\right)_{\bmod 2}\right| \leqslant \frac{5}{8} .
$$

Further,

$$
\left|\frac{\operatorname{lm}(E+e)}{n(l+m)}\right|<\frac{1}{9(17+12 \rho)}\left[\frac{3}{16}(1+12 \rho)+3\right]=\frac{1}{48},
$$

so

$$
d\left(\frac{r+e}{1 / l+1 / m}\right) \geqslant\left[1-\cos \pi\left(\frac{1}{3}-\frac{1}{48}\right)\right]\left[\cos \pi\left(\frac{1}{3}+\frac{1}{48}\right)-\cos \frac{3 \pi}{8}\right]=d_{1}>0 .
$$


Thus, in (I)-(IV) we have shown that, if

$$
\frac{l \rho}{m} \geqslant \frac{1}{12} \text { and } \quad \frac{l m}{n(l+m)}<\frac{1}{9(17+12 \rho)},
$$

there exists $x \in R$ such that $d(x) \geqslant \min \left(d_{1}, d_{2}\right)=d_{3}>0$. Now from

$$
|\cos (x+\delta x)-\cos x|=2\left|\sin \left(x+\frac{\delta x}{2}\right) \sin \frac{\delta x}{2}\right| \leqslant|\delta x|,
$$

we deduce that

$$
|d(x+\delta x)-d(x)| \leqslant 12 \pi|\delta x| / l .
$$

Since we may find $s,(s, 2 \operatorname{lmn})=1$ within $\rho / 2$ of $x$, it follows that $d(s)>0$ if $6 \pi \rho / l<d_{3}$ and the above conditions are satisfied. Using the initial conditions, we have, for sufficiently large $n$,

$$
\frac{l m}{n(l+m)} \leqslant \sqrt{\frac{\rho}{2 n}}<\frac{1}{9(17+12 \rho)} .
$$

Thus the proof of Case II is completed if $c=d_{3} / 6 \pi$.

Case III. $\operatorname{lm}^{2} \rho<n$.

If $s \equiv 1(\bmod 2 l m)$ then, from $(4)$,

$$
\begin{gathered}
d(s)=-4 \cos \frac{\pi}{2}\left(-\frac{1}{l}+\frac{1}{m}+\frac{s}{n}\right) \cos \frac{\pi}{2}\left(\frac{1}{l}-\frac{1}{m}-\frac{s}{n}\right) \\
\cdot \cos \frac{\pi}{2}\left(\frac{1}{l}+\frac{1}{m}-\frac{s}{n}\right) \cos \frac{\pi}{2}\left(\frac{1}{l}+\frac{1}{m}+\frac{s}{n}\right) .
\end{gathered}
$$

Let $s$ be the largest such integer which is relatively prime to $n$ and for which $1 / l+1 / m+s / n<1$, and let $s^{\prime}$ be the next largest such integer. Then $d\left(s^{\prime}\right)>0$ if

$$
\frac{1}{l}+\frac{1}{m}-\frac{s+2 \operatorname{lm} \rho}{n}>-\left(\frac{1}{l}+\frac{1}{m}+\frac{s}{n}\right), \quad \frac{1}{l}-\frac{1}{m}+\frac{s+2 \operatorname{lm} \rho}{n}<\frac{1}{l}+\frac{1}{m}+\frac{s}{n} .
$$

Since both of these are implied by $\operatorname{lm}^{2} \rho<n$, the result follows.

Case IV. $l \rho / m<1 / 12$.

Let $(m, n)=r, m=m^{\prime} r, n=n^{\prime} r$. By the Chinese Remainder Theorem there are rational solutions to:

(I) $x / m \equiv \frac{1}{2}(\bmod 2), x / n \equiv \frac{1}{2}(\bmod 2)$ if $m^{\prime} \equiv n^{\prime}(\bmod 4)$.

(II) $x / m \equiv \frac{1}{2}(\bmod 2), x / n \equiv-\frac{1}{2}(\bmod 2)$ if $m^{\prime} \equiv n^{\prime}+2(\bmod 4)$.

(III) $x / m \equiv \frac{1}{2} \pm \varepsilon / m^{\prime}(\bmod 2), x / n \equiv \frac{1}{2} \pm \delta / n^{\prime}(\bmod 2)$ for any $\varepsilon, \delta \in Q$ such that $\varepsilon+\delta=\frac{1}{2}$ if $m^{\prime} \equiv n^{\prime} \pm 1(\bmod 4)$.

The proof now splits into subcases depending on the congruence class of $l \bmod 4$.

(a) $l \equiv 1(\bmod 4)$.

Now $((l+1) / 2,2 l)=1$ and so we can choose $s \equiv(l+1) / 2(\bmod 2 l),(s, 2 \operatorname{lmn})$ $=1$ within $l \rho$ of $x$, where $x$ is a solution of the equations in (I), (II) or (III). For (I), $d(s)>0$ if

$$
l \rho\left(\frac{1}{m}+\frac{1}{n}\right)<\frac{1}{2}+\frac{1}{2 l} \text { and } \quad l \rho\left(\frac{1}{m}-\frac{1}{n}\right)<\frac{1}{2}-\frac{1}{2 l}
$$

by (5), and these hold if $l \rho / m<1 / 4$. Likewise for (II), $d(s)>0$ if

$$
l \rho\left(\frac{1}{m}+\frac{1}{n}\right)<\frac{1}{2}-\frac{1}{2 l},
$$


which holds for $l \rho / m<1 / 5$. In (III) take $\varepsilon=0, \delta=\frac{1}{2}$, so that $d(s)>0$ if

$$
\frac{1}{2 n^{\prime}}+l \rho\left(\frac{1}{m}-\frac{1}{n}\right)<\frac{1}{2}-\frac{1}{2 l} \text { and } \frac{1}{2 n^{\prime}}+l \rho\left(\frac{1}{m}+\frac{1}{n}\right)<\frac{1}{2}+\frac{1}{2 l} \text {. }
$$

Since $n^{\prime} \geqslant 2$, these are both satisfied for $l \rho / m<1 / 8$.

(b) $l \equiv 2(\bmod 4)$.

For $l \neq 2$ in Cases (I) and (II), choose $s \equiv l / 2+2(\bmod 2 l),(s, 2 l m n)=1$ within $l \rho$ of $x$, and the result follows as in (a). In Case (III) let $\varepsilon=m^{\prime} / 2\left(m^{\prime}+n^{\prime}\right)$, $\delta=n^{\prime} / 2\left(m^{\prime}+n^{\prime}\right)$ and choose $s \equiv l / 2-2(\bmod 2 l),(s, 2 l m n)=1$ within $l \rho$ of $x$, in which case $d(s)>0$ if

$$
\frac{1}{m^{\prime}+n^{\prime}}+l \rho\left(\frac{1}{m}-\frac{1}{n}\right)<\frac{1}{2}+\frac{2}{l} \text { and } l \rho\left(\frac{1}{m}+\frac{1}{m}\right)<\frac{1}{2}-\frac{2}{l}
$$

which are valid if $l \rho / m<1 / 12$. For $l=2$ we can find an $s,(s, 2 m n)=1$ within $\rho / 2$ of any given $x$, and the result follows as above.

(c) $l \equiv-1(\bmod 4)$.

Choosing $s \equiv(l-1) / 2(\bmod 2 l),(s, 2 l m n)=1$ within $l \rho$ of $x$ a solution of (I)-(III), with, in Case (III), $\varepsilon=m^{\prime} / 2\left(m^{\prime}+n^{\prime}\right), \delta=n^{\prime} / 2\left(m^{\prime}+n^{\prime}\right)$, the result follows as before by checking the relevant inequalities.

(d) $l \equiv 0(\bmod 4)$.

The same method works with $\varepsilon, \delta$ as in $(\mathrm{c})$ and $s \equiv l / 2-1(\bmod 2 l)$. Case IV is thus completed.

It remains to show that Cases I-IV are sufficient to prove our theorem. Cases I, II and IV suffice unless $l<\rho / c$ and $m<12 \rho \cdot(\rho / c)$, in which case

$$
\operatorname{lm}^{2} \rho<\frac{\rho}{c}\left(12 \rho^{2} / c\right)^{2} \rho=144 \rho^{6} / c^{3}<n
$$

for sufficiently large $n$. Thus Case III completes the proof.

5. The proof of Theorem 4 can provide explicit bounds for the number of exceptional triples $(l, m, n)$ but, because of the slow rate of growth of $\log \log x$, these bounds are hopelessly large. The best we can do is determine all exceptional triples within certain special families of triples.

TheOREM 5. $\sigma=(2, m, n)$ has a representative in $Q\left(\cos \frac{\pi}{m}, \cos \frac{\pi}{n}\right)$ if and only if $(m, n)=(4,6),(6,6),(6,10)$.

Proof. Let $m=2^{k_{1}} m^{\prime}, n=2^{k_{1}+k_{2}} n^{\prime}$, where $m^{\prime}, n^{\prime}$ are odd and $k_{1}, k_{2} \geqslant 0$.

We consider various cases:

A. $k_{2}=0$.

Let $s=2^{k_{1}-1} m^{\prime} n^{\prime}+\mathscr{E}$, when $\mathscr{E} \in Q$ is chosen so that $(s, 2 m n)=1$. So

$$
d(s)=\sin ^{2} \pi\left(\frac{n^{\prime}}{2}+\frac{\mathscr{E}}{m}\right)-\cos ^{2} \pi\left(\frac{m^{\prime}}{2}+\frac{\mathscr{E}}{n}\right)=\cos ^{2} \frac{\mathscr{E} \pi}{m}-\sin ^{2} \frac{\mathscr{E} \pi}{n}
$$

(a) $k_{1}=0$. Let $\mathscr{E}= \pm \frac{1}{2}$. Then $d(s)>0$.

(b) $k_{1}=1$. Let $\mathscr{E}=2$. Then $d(s)>0$ unless $(m, n)=(6,6)$ or $(6,10)$.

(c) $k_{1}>1$. Let $\mathscr{E}=+1$. Then $d(s)>0$. 
B. $k_{2}>0$.

Choose $t \in Z$ such that $m^{\prime} t \equiv 2^{k_{2}}-1\left(\bmod 2^{k_{2}+2}\right)$ and let $s=t 2^{k_{1}-1} m^{\prime} n^{\prime}+\mathscr{E}^{\prime}$ to give

$$
\begin{aligned}
d(s) & =\sin ^{2} \pi\left(\frac{t n^{\prime}}{2}+\frac{\mathscr{E}^{\prime}}{m}\right)-\cos ^{2} \pi\left(\frac{1}{2}-\frac{1}{2^{k_{2}+1}}+\frac{\mathscr{E}^{\prime}}{n}\right) \\
& =\cos ^{2} \frac{\mathscr{E}^{\prime} \pi}{m}-\cos ^{2} \pi\left(\frac{1}{2}-\frac{1}{2^{k_{2}+1}}+\frac{\mathscr{E}^{\prime}}{n}\right) .
\end{aligned}
$$

(a) $k_{1}=0, \mathscr{E}= \pm \frac{1}{2}$. Then $d(s)>0$ if $1 / 2 m<1 / 2-1 / 2^{k_{2}+1}-1 / 2 n$, which holds since $1 / m+1 / n<1 / 2$.

(b) $k_{1}=1, \mathscr{E}=2$. Then $d(s)>0$ if $2 / m<1 / 2-\left|1 / 2^{k_{2}+1}-2 / n\right|$, which holds for $m>6$.

For $m=6$ let $s=3 n / 2+1$ so that $d(s)=\sin ^{2} \frac{\pi}{6}-\sin ^{2} \frac{\pi}{n}>0$ unless $n=4$.

(c) $k_{1}>1, \mathscr{E}=1$. Then $d(s)>0$.

It remains to show that the exceptions $(2,4,6),(2,6,6),(2,6,10)$ do indeed have representatives in the minimal field. From Theorem 2 we must consider the equation $x^{2}+y^{2}=-d$. For $(2,4,6)$ and $(2,6,6), d=-\frac{1}{4}$ and $-\frac{1}{2}$, respectively, and the equations have solutions in $Q$. For $(2,6,10)$,

$$
d=-\left(\cos ^{2} \frac{\pi}{10}-\frac{1}{4}\right)=-\left(\frac{1+\sqrt{5}}{4}\right)^{2},
$$

so the equation has a solution in

$$
Q(\sqrt{5})<Q\left(\cos \frac{\pi}{6}, \cos \frac{\pi}{10}\right)
$$

THEOREM 6. If $\sigma=(l, m, n)$ with $l, m, n$ odd, then $\sigma$ does not have a representative in $Q\left(\cos \frac{\pi}{l}, \cos \frac{\pi}{m}, \cos \frac{\pi}{n}\right)$.

Proof. Let $s=(\operatorname{lm} n \pm 1) / 2$, where the sign is chosen so that $(s, 2 \operatorname{lm} n)=1$; then

$$
\begin{aligned}
d(s) & \geqslant 1-\sin ^{2} \frac{\pi}{2 l}-\sin ^{2} \frac{\pi}{2 m}-\sin ^{2} \frac{\pi}{2 n}-2 \sin \frac{\pi}{2 l} \sin \frac{\pi}{2 m} \sin \frac{\pi}{2 n} \\
& >1-3 \sin ^{2} \frac{\pi}{6}-2 \sin ^{3} \frac{\pi}{6}=0 .
\end{aligned}
$$

REMARK. A more complicated argument yields that if $\sigma=(l, m, n)$, where $l=2^{\alpha} l^{\prime}$, $m=2^{\alpha} m^{\prime}, n=2^{\alpha} n^{\prime}$ with $l^{\prime}, m^{\prime}, n^{\prime}$ odd and $l, m, n>2$, then $\sigma$ does not have a representative in $Q\left(\cos \frac{\pi}{l}, \cos \frac{\pi}{m}, \cos \frac{\pi}{n}\right)$ (see [17]).

Finally, for this section, we list the known "exceptional triples", i.e. triples $(l, m, n)$ for which there does not exist a Galois automorphism $\sigma$ of $Q\left(\cos \frac{\pi}{l}, \cos \frac{\pi}{m}, \cos \frac{\pi}{n}\right) \mid Q$ with $\sigma(d)>0$ :

$$
\begin{array}{lll}
(2,4,6) & (2,6,6) & (2,6,10) \\
(3,4,4) & (3,6,6) & (3,10,10) \\
(4,6,12) & \\
(5,6,6) & \\
(6,9,18) & (6,10,15) & \\
(14,21,42) &
\end{array}
$$


6. As mentioned in $\S 1, \operatorname{PSL}(2, R)$ can be identified with $\operatorname{PGL}_{0}(2, R)$. Extending the definition of $\S 2$, the signature $\sigma$ is said to have a PGL-representative in $F$ if there exists a Fuchsian group $\Gamma$ with signature $\sigma$ such that $\Gamma<\operatorname{PGL}_{0}(2, F)=\operatorname{PGL}(2, F)$ $\cap \mathrm{PGL}_{0}(2, R)$. Let $P$ denote the natural map: $\operatorname{GL}(2, R) \rightarrow \operatorname{PGL}(2, R)$.

Any element of $\mathrm{PGL}_{0}(2, F)$ is $\mathrm{PGL}_{0}(2, F)$ conjugate to

$$
P\left(\begin{array}{ll}
0 & \lambda \\
-1 / \lambda & \tau
\end{array}\right)
$$

Thus there is an $x \in R-\{0\}$ such that $\lambda x,-x / \lambda, \tau x \in F$, so $\lambda \tau, \tau^{2}, \lambda^{2}$ must lie in $F$. Thus if $\sigma=\left(g ; m_{1}, m_{2}, \ldots, m_{r} ; s ; t\right)$ then $F$ must contain

$$
Q\left(\cos ^{2} \frac{\pi}{m_{1}}, \cos ^{2} \frac{\pi}{m_{2}}, \ldots, \cos ^{2} \frac{\pi}{m_{r}}\right) .
$$

If $\tau=2 \cos \left(\pi / m_{i}\right)$ then $x=2 \cos \left(\pi / m_{i}\right) x_{i}$, where $x_{i} \in F$. Note that any parabolic element of $\Gamma$ lying in $\operatorname{PGL}(2, F)$ actually lies in $\operatorname{PSL}(2, F)$, and the commutator of two elements of $\operatorname{PGL}(2, F)$ also lies in $\operatorname{PSL}(2, F)$. Thus if $t=0$ in the signature $\sigma$ and $\Gamma<\operatorname{PGL}(2, F)$, then from the long relation in (1), the product of the elliptic elements must lie in $\operatorname{PSL}(2, F)$. Thus, with notation as above,

$$
\prod_{i=1}^{v} \cos ^{2}\left(\frac{\pi}{m_{i}}\right) x_{i}^{2}=1
$$

so $\cos \left(\pi / m_{1}\right), \cos \left(\pi / m_{2}\right), \ldots, \cos \left(\pi / m_{r}\right)$ must also lie in the minimal field. If $\mathscr{T}_{F}^{*}(\Gamma)$ denotes the subset of $\mathscr{T}(\Gamma)$ corresponding to groups whose elements lie in $\mathrm{PGL}_{0}(2, F)$, then a variation of Takeuchi's argument gives

THEOREM 7. If $\Gamma$ has signature $\sigma=\left(g ; m_{1}, m_{2}, \ldots, m_{r} ; s ; t\right)$ and is not a cocompact triangle group, then $\mathscr{T}_{F}(\Gamma)$ is dense in $(\Gamma)$ when

$$
F=\left[\begin{array}{ll}
Q\left(\cos ^{2} \frac{\pi}{m_{1}}, \ldots, \cos ^{2} \frac{\pi}{m_{r}}\right), & t>0, \\
Q\left(\cos ^{2} \frac{\pi}{m_{1}}, \ldots, \cos ^{2} \frac{\pi}{m_{2}}, \cos \frac{\pi}{m_{1}} \cdots \cos \frac{\pi}{m_{r}}\right), & t=0 .
\end{array}\right.
$$

Likewise, extending Theorem 2, we obtain

THEOREM 8. $\sigma=(l, m, n)$ has a PGL-representative in $F$ where

$$
Q\left(\cos ^{2} \frac{\pi}{l}, \cos ^{2} \frac{\pi}{m}, \cos ^{2} \frac{\pi}{n}, \cos \frac{\pi}{l} \cos \frac{\pi}{m} \cos \frac{\pi}{n}\right)<F<R
$$

if and only if there is a solution in $F$ of the equation

$$
x^{2} \cos ^{2} \frac{\pi}{m}\left(1-\cos ^{2} \frac{\pi}{n}\right)+y^{2} \cos ^{2} \frac{\pi}{m} \cos ^{2} \frac{\pi}{n}=-d
$$

where $l \leqslant m \leqslant n$.

From this it follows that we can always find a PGL-representative at least in a quadratic extension of the minimal PGL field described in Theorem 8. Furthermore, since this minimal PGL field is a subfield of the minimal PSL field, Theorems 8 and 4 yield

THEOREM 9. Except for finitely many triples $(l, m, n), \sigma=(l, m, n)$ does not have a PGL-representative in

$$
Q\left(\cos ^{2} \frac{\pi}{l}, \cos ^{2} \frac{\pi}{m}, \cos ^{2} \frac{\pi}{n}, \cos \frac{\pi}{l} \cos \frac{\pi}{m} \cos \frac{\pi}{n}\right)
$$


REMARK 1. Theorems 5 and 8 show that for the special family of signature $\sigma=(2, m, n)$ only $(2,4,6),(2,6,6)(2,6,10)$ could have PGL-representatives in the minimal field. However, in all three cases it can be shown, using Theorem 8 , that they do not have PGL-representatives in the minimal field.

REMARK 2. In [5-7] Greenberg investigated homomorphisms of triangle groups into $\operatorname{PSL}(2, C)$ and showed that if there was a Galois automorphism $\sigma$ of $Q\left(\cos \frac{\pi}{l}, \cos \frac{\pi}{m}, \cos \frac{\pi}{n}\right)$ such that $\sigma(d)>0$, then there was an embedding of an $(l, m, n)$ triangle group into $\operatorname{PSU}(2, C)$. Thus from Theorem 4 , we deduce

THEOREM 10. For all but a finite number of exceptions, every $(l, m, n)$ triangle group can be embedded in $\mathrm{PSU}(2, C)$.

\section{REFERENCES}

1. L. Bers, On boundaries of Teichmüller spaces and on Kleinian groups. I, Ann. of Math. (2) 91 (1970), $570-600$.

2. A. L. Edmonds, J. H. Ewing and R. S. Kulkarni, Torsion free subgroups of Fuchsian groups and tessellations of surfaces, Invent. Math. 69 (1982), 331-346.

3. , Regular tessellations of surfaces and ( $p, q, 2)$-triangle groups, Ann. of Math. (2) 116 (1982), $113-132$.

4. A. L. Edmonds, R. S. Kulkarni and R. E. Stong, Realizability of branched coverings of surfaces (preprint).

5. L. Greenberg, Homomorphisms of triangle groups into PSL(2, C), Ann. of Math. Stud., vol. 97, Princeton Univ. Press, Princeton, N.J., 1981, pp. 167-182.

6. Homomorphisms of triangle groups into $\mathrm{SO}(3)$, Modular Functions in Analysis and Number Theory, (T. Metzer, ed.), Univ. of Pittsburgh, 1983.

7. Homomorphisms and quadratic differentials of triangle groups (unpublished).

8. G. H. Hardy and E. M. Wright, An introduction to the theory of numbers, 5th ed., Oxford, 1979.

9. A. W. Knapp, Doubly generated Fuchsian groups, Michigan Math. J. 15 (1968), 289-304.

10. R. S. Kulkarni, An extension of a theorem of Kurosh and applications to Fuchsian groups (preprint).

11. J. Lehner and M. Newman, Real 2-dimensional representations of the modular group and related groups, Amer. J. Math. 87 (1965), 945-954.

12. , Real 2-dimensional representations of the free product of two finite cyclic groups, Proc. Cambridge Philos. Soc. 62 (1966), 135-141.

13. W. Magnus, Rational representations of Fuchsian groups and non-parabolic subgroups of the modular group, Nachr. Akad. Wiss. Göttingen II: Math. Phys. K1. 1973, 179-189.

14. J. Mennicke, A note on regular coverings of closed orientable surfaces, Proc. Glasgow Math. Assoc. 5 (1961), 49-66.

15. M. H. Millington, Subgroups of the classical modular group, J. London Math. Soc. (2) 1 (1969), $351-357$.

16. K. Takeuchi, Fuchsian groups contained in SL(2, Q), J. Math. Soc. Japan 23 (1971), 82-94.

17. P. L. Waterman, Fuchsian groups and algebraic number fields, Thesis, Aberdeen, 1982.

18. A. Weil, On discrete subgroups of Lie groups, Ann. of Math. (2) 72 (1960), 369-384.

Department of Mathematics, Temple University, Philadelphia, Pennsylvania 19122

Current address (P. L. Waterman): Department of Mathematics, Northern Illinois University, DeKalb, Illinois 60115

Current address (C. Maclachlan): Department of Mathematics, University of Aberdeen, Aberdeen, AB9 2TY, Scotland 